\title{
Defects in adenosine metabolism identified in ALS
}

"The CSF analysis reproduced what we found using PET and suggests that NREM SWA declines immediately before or after the onset of cognitive symptoms due to $\mathrm{AD}$, but longitudinal studies are needed to establish the exact timing of changes in sleep with $\mathrm{AD}$ pathology and symptoms," notes Lucey. "A major implication of this study is that measuring NREM SWA might represent a noninvasive and inexpensive way to screen for risk of cognitive decline caused by $\mathrm{AD}$, which would be helpful in future clinical trials and possibly screening in the clinic."

\section{The CSF analysis reproduced what we found using PET

this protein has already successfully completed a phase II clinical trial
Rebecca Kelsey

ORIGINAL ARTICLE Lucey, B. P. et al.

Reduced non-rapid eye movement sleep is associated with tau pathology in early Alzheimer's disease. Sci. Transl Med. https://doi.org/10.1126/ scitranslmed.aau6550 (2019) FURTHER READING Ju, Y. E. et al. Sleep and Alzheimer disease pathology - a bidirectional relationship. Nat. Rev. Neurol. 10, 115-119 (2014)

"To our surprise, we also found greatly reduced $A \beta$ pathology and reductions in generation of $A \beta$ peptides, mediated via blockade of the NF-кB-BACE1 pathway," says Zlokovic. This pathway leads to production of $A \beta$, which aggregates in the brain in AD.

The findings indicate that $3 \mathrm{~K} 3 \mathrm{~A}-\mathrm{APC}$ provides an opportunity to use a single drug to prevent $A \beta$ production in addition to providing neuronal and vascular protection and mitigating inflammation.

"What is particularly attractive with $3 \mathrm{~K} 3 \mathrm{~A}-\mathrm{APC}$ is the fact that this protein has already successfully completed a phase II clinical trial and has shown clear target engagement in the living human brain," explains Zlokovic. "We are now considering a proof-of-concept study in patients with early AD to show target engagement by monitoring cerebrospinal fluid and imaging biomarkers and with cognitive tests." lan Fyfe

ORIGINAL ARTICLE Lazic, D. et al.

3K3A-activated protein C blocks amyloidogenic BACE1 pathway and improves functional outcome in mice.J. Exp. Med. https://doi.org/ 10.1084/jem.20181035 (2019)
Patients with amyotrophic lateral sclerosis (ALS) - a motor neuron disease (MND) characterized by degeneration of upper and lower motor neurons exhibit dysfunctional energy metabolism, which is known to contribute to disease progression. In a new study published in Brain, Scott Allen and colleagues have pinpointed a specific ALS-associated defect in astrocytic adenosine metabolism that could be amenable to therapeutic intervention.

"We previously found that in patients with ALS, skin cells showed similar mitochondrial defects to CNS cells, such as neurons and astrocytes," explains Allen. "However, unlike CNS cells, skin cells could upregulate their glycolytic pathways to maintain energy levels, and this started me thinking about the intrinsic ability of different cell types to mobilize alternative energy supplies to cope with bioenergetic stress - a property that I have termed 'metabolic flexibility'."

To investigate the phenomenon of metabolic flexibility in ALS, Allen and colleagues obtained fibroblasts from six patients with C9orf72-related ALS, three patients with sporadic ALS and eight healthy controls. The researchers reprogrammed these cells to generate induced neuronal progenitor cells (iNPCs), which were subsequently differentiated into astrocytes. The iNPC-derived astrocytes were then subjected to a novel metabolic screen, which tested the ability of the cells to generate NADH from an array of 91 different energy substrates.

The iNPC-derived astrocytes from the patients with ALS showed an impaired capacity to generate NADH in the presence of adenosine. This defect was attributed to a reduction in the activity of adenosine deaminase, an enzyme that converts adenosine to inosine.

"This is the first time that adenosine deaminase has been implicated in MND," points out Allen. "The consequence of this loss of enzyme activity under conditions of neurodegeneration would be a toxic build-up of adenosine in the CNS and subsequent loss of production of inosine, a metabolic intermediate that is generally protective."

Allen and his co-workers found that inosine supplementation improved the survival of motor neurons in co-culture with iNPC-derived astrocytes from patients with ALS. This finding indicates that conversion of adenosine to inosine in astrocytes contributes to the metabolic support of neurons. The researchers propose that increasing adenosine deaminase levels, in combination with inosine supplementation, could provide a new approach to slowing disease progression in patients with ALS.

"Altering the level of adenosine deaminase by gene therapy has been shown to be beneficial and safe in patients with severe combined immunodeficiency disease, and inosine is a safe and readily available nutritional supplement that has been successfully tested in patients with Parkinson disease," comments Allen. "There is hope, therefore, that in the future, this combination of treatment could work in people with ALS, improving their quality of life and helping them to live longer."

Heather Wood

ORIGINAL ARTICLE Allen, S. P. et al. Astrocyte adenosine deaminase loss increases motor neuron toxicity in amyotrophic lateral sclerosis. Brain https://doi.org/10.1093/brain/awy353 (2019) FURTHER READING Allen, S. P. et al. Superoxide dismutase 1 mutation in a cellular model of amyotrophic lateral sclerosis shifts energy generation from oxidative phosphorylation to glycolysis. Neurobiol. Aging 35 , 1499-1509 (2014) 